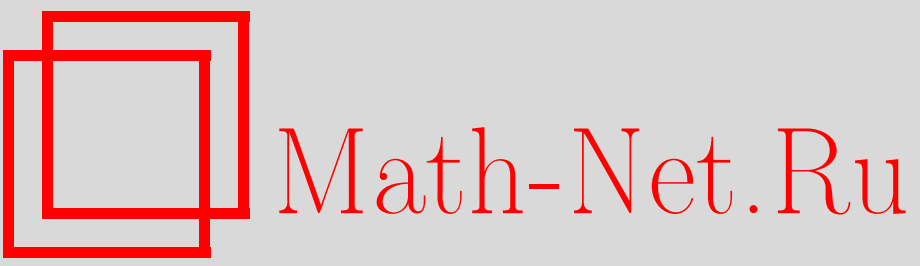

М. А. Заусаева, В. Е. Зотеев, Определение параметров двумерных динамических процессов на основе разностных схем, Вестн. Сам. гос. техн. ун-та. Сер. Физ.мат. науки, 2010, выпуск 1(), 154-161

DOI: https://doi.org/10.14498/vsgtu781

Использование Общероссийского математического портала Math-Net.Ru подразумевает, что вы прочитали и согласны с пользовательским соглашением

http: //www. mathnet.ru/rus/agreement

Параметры загрузки:

IP : 54.172 .240 .79

26 апреля 2023 г., $14: 46: 34$ 


\title{
УДК 681.5.015 \\ ОПРЕДЕЛЕНИЕ ПАРАМЕТРОВ ДВУМЕРНЫХ ДИНАМИЧЕСКИХ ПРОЦЕССОВ НА ОСНОВЕ РАЗНОСТНЫХ СХЕМ
}

\author{
M. А. Заусаева, В. Е. Зотеев
}

Самарский государственный технический университет, 443100, Самара, ул. Молодогвардейская, 244.

E-mail: zausmasha@mail.ru

Рассматривается построение разностных схем, описывающих результаты наблюдений двумерных пространственно-временных функииональных зависимостей, и численный метод определения параметров таких зависимостей на основе разностных схем. Алгоритм метода включает итерационную процедуру среднеквадратичного оценивания коэфбициентов линейно-параметрической дискретной модели в форме стохастических разностных уравнений. Такой подход к решению задачи идентибикации двумерных пространственно-временных функииональных зависимостей позволяет обеспечить высокую адекватность построенной модели и, как следствие, добиться высокой точности результатов оценивания параметров модели.

Ключевые слова: параметрическая идентификащия, разностные схемы, среднеквадратичное приближение.

Проблема достоверной оценки параметров математической модели по экспериментальным данным является важнейшей проблемой для различных областей науки и техники. Одним из эффективных методов параметрической идентификации динамических систем является метод, в основе которого лежат линейно-параметрические дискретные модели, описывающие в форме разностных уравнений результаты наблюдений [1]. Однако область применения таких моделей ограничена одномерными динамическими процессами, при описании которых используются нелинейные функциональные зависимости от одной временной переменной. Вместе с тем в практике эксперимента при моделировании объектов и явлений различной физической природы широко используется аппарат математической физики, основу которого составляют дифференциальные уравнения в частных производных. Решения таких уравнений, как правило, представляют собой пространственно-временные функциональные зависимости, например, двумерные зависимости в задачах теплопроводности, описывающие изменение температуры стержневой конструкции по её длине. Таким образом, становится актуальной задача разработки и исследования новых линейно-параметрических дискретных моделей, описывающих в форме разностных схем многомерные динамические процессы. В данной работе представлены результаты решения этой задачи для двумерного динамического процесса, который может быть описан в виде произведения двух мультипликативных компонент, каждая из которых связана только с одной из двух независимых переменных.

Пусть динамический процесс описывается функцией двух независимых

Мария Анатольевна Заусаева, аспирант, каф. прикладной математики и информатики. Владимир Евгенъевич Зотеев (к.ф.-м.н., доц.), доцент, каф. прикладной математики и информатики. 
переменных:

$$
\tilde{z}(t, x)=y(t) u(x) .
$$

определенной и непрерывной в пространственно-временной области $0 \leqslant x \leqslant l$, $0 \leqslant t \leqslant T$, где $y(t)=c_{1}\left(1-e^{-\alpha_{1} t}\right)+c_{2}\left(1-e^{-\alpha_{2} t}\right), u(x)=e^{\beta x}, c_{1}, c_{2}, \alpha_{1}, \alpha_{2}$, $\beta \in \mathbb{R}$.

Рассмотрим задачу оценки параметров функциональной зависимости (1) по экспериментальным данным $z_{k, j}=\tilde{z}(\tau k, j h)+\varepsilon_{k, j}$, полученным в узлах конечно-разностной сетки $\omega_{\tau, h}$ :

$$
\omega_{\tau, h}=\left\{t_{k}=k \tau, k \equiv 0,1, \ldots N-1 ; x_{j}=j h, j \equiv 0,1, \ldots, M-1\right\},
$$

где $\tau=T /(N-1)$ - период дискретизации по временной координате; $h=$ $=l /(M-1)$ - шаг дискретизации по независимой переменной $x ; \varepsilon_{k, j}$-случайный разброс (случайная помеха) в результатах наблюдений; $N$ и $M$ - число точек по координатам $t$ и $x$ соответственно. Значения $\varepsilon_{k, j}$ некоррелированны (взаимно независимы), имеют нулевое математическое ожидание и одинаковые дисперсии. В основе решения этой задачи лежит построение и исследование линейно-параметрической дискретной модели, описывающей в форме разностной схемы дискретные значения функциональной зависимости (1). Для формирования этой модели рассмотрим построение разностных уравнений для каждой мультипликативной составляющей в отдельности.

Временная последовательность дискретных значений функции $y(t)$ описывается выражением вида

$$
y_{k}=c_{1}\left(1-e^{-\alpha_{1} \tau k}\right)+c_{2}\left(1-e^{-\alpha_{2} \tau k}\right)
$$

или

$$
y_{k}-c_{1}-c_{2}=-c_{1} e^{-\alpha_{1} \tau k}-c_{2} e^{-\alpha_{2} \tau k}
$$

Отсюда имеем

$$
\begin{aligned}
& y_{k-1}-c_{1}-c_{2}=-c_{1} e^{-\alpha_{1} \tau k} \mu_{1}-c_{2} e^{-\alpha_{2} \tau k} \mu_{2}, \\
& y_{k-2}-c_{1}-c_{2}=-c_{1} e^{-\alpha_{1} \tau k} \mu_{1}^{2}-c_{2} e^{-\alpha_{2} \tau k} \mu_{2}^{2},
\end{aligned}
$$

где $\mu_{1}=e^{\alpha_{1} \tau}$ и $\mu_{2}=e^{\alpha_{2} \tau}$. Выделяя из этих двух уравнений выражения $-c_{1} e^{-\alpha_{1} \tau k}$ и $-c_{2} e^{-\alpha_{2} \tau k}$ и подставляя их в $(2)$, получаем разностное уравнение вида

$$
y_{k}=\lambda_{1} y_{k-1}+\lambda_{2} y_{k-2}+\lambda_{3} \quad(k \equiv 2,3, \ldots, N-1),
$$

где

$$
\lambda_{1}=\frac{\mu_{1}+\mu_{2}}{\mu_{1} \mu_{2}}, \quad \lambda_{2}=-\frac{1}{\mu_{1} \mu_{2}}, \quad \lambda_{3}=\left(c_{1}+c_{2}\right)\left(1-\lambda_{1}-\lambda_{2}\right) .
$$

Последовательность дискретных значений функции $u(x)$ с шагом $h$ описывается выражением вида $u_{j}=e^{j \beta h}$. Так как $u_{j-1}=e^{j \beta h} e^{-\beta h}$, то очевидно, что $u_{j}=e^{\beta h} u_{j-1}$ или

$$
u_{j}=\lambda_{4} u_{j-1}, \quad j \equiv 1,2, \ldots, M-1,
$$

где $\lambda_{4}=e^{\beta h}$. 
Множество дискретных значений функции $(1)$ в узлах сетки $\omega_{\tau, h}$ описывается выражением

$$
\tilde{z}_{k, j}=y_{k} u_{j}, \quad k \equiv 0,1,2, \ldots, N-1, \quad j \equiv 0,1,2, \ldots, M-1 .
$$

Тогда разностное уравнение (3) можно представить в виде

$$
\frac{\tilde{z}_{k, j}}{u_{j}}=\lambda_{1} \frac{\tilde{z}_{k-1}}{u_{j}}+\lambda_{2} \frac{\tilde{z}_{k-2, j}}{u_{j}}+\lambda_{3}
$$

Отсюда имеем

$$
\tilde{z}_{k, j}=\lambda_{1} \tilde{z}_{k-1, j}+\lambda_{2} \tilde{z}_{k-2, j}+\lambda_{3} u_{j}
$$

и, следовательно,

$$
\tilde{z}_{k, j-1}=\lambda_{1} \tilde{z}_{k-1, j-1}+\lambda_{2} \tilde{z}_{k-2, j-1}+\lambda_{3} u_{j-1}
$$

С учётом равенства (5) первое выражение можно преобразовать к виду

$$
\tilde{z}_{k, j}=\lambda_{1} \tilde{z}_{k-1, j}+\lambda_{2} \tilde{z}_{k-2, j}+\lambda_{3} \lambda_{4} u_{j-1} .
$$

Если теперь обе части второго выражения умножить на $\lambda_{4}$, то получим два уравнения, из которых, исключая слагаемое $\lambda_{3} \lambda_{4} u_{j-1}$, можно сформировать разностную схему вида

$$
\begin{gathered}
\tilde{z}_{k, j}=\lambda_{1} \tilde{z}_{k-1, j}+\lambda_{2} \tilde{z}_{k-2, j}+\lambda_{4} \tilde{z}_{k, j-1}+\lambda_{5} \tilde{z}_{k-1, j-1}+\lambda_{6} \tilde{z}_{k-2, j-1} \\
(k \equiv 2,3, \ldots, N-1, j \equiv 1,2, \ldots, M-1)
\end{gathered}
$$

где $\lambda_{5}=-\lambda_{1} \lambda_{4}, \lambda_{6}=-\lambda_{2} \lambda_{4}$.

На рис. 1 закрашенными узлами представлена геометрическая интерпретация построенной разностной схемы $(6)$ на конечно-разностной сетке $\omega_{\tau, h}$ (шаблон разностной схемы). Остальные значения разностной схемы (незакрашенные узлы на шаблоне) описываются уравнениями, которые можно получить непосредственно из функциональной зависимости (1) для узлов, соответствующих $k=0,1$ и $j=0$ :

$$
\begin{gathered}
\tilde{z}_{0,0}=0, \quad \tilde{z}_{1,0}=\lambda_{7}, \\
\tilde{z}_{k, 0}=\lambda_{1} \tilde{z}_{k-1,0}+\lambda_{2} \tilde{z}_{k-2,0}+\lambda_{3}(k \equiv 2,3, \ldots, N-1), \\
\tilde{z}_{k, j}=\lambda_{4} \tilde{z}_{k, j-1} \quad(k \equiv 0,1 ; j \equiv 1,2, \ldots, M-1),
\end{gathered}
$$

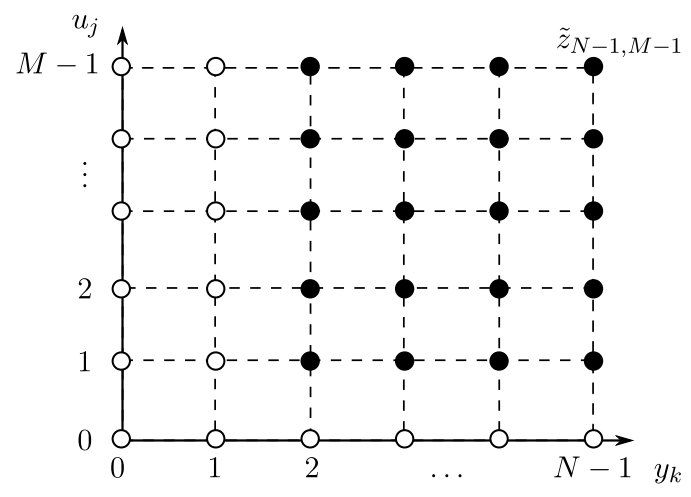

Рис. 1. Шаблон разностной схемы $\omega_{\tau, h}$ для двумерной пространственно-временной функциональной зависимости 
где $\lambda_{7}=c_{1}\left(1-\frac{1}{\mu_{1}}\right)+c_{2}\left(1-\frac{1}{\mu_{2}}\right)$.

При обработке экспериментальных данных формируется выборка результатов наблюдений объёма $V=M N$ :

$$
z_{k, j}=\tilde{z}_{k, j}+\varepsilon_{k, j} \quad(k \equiv 0,1, \ldots, N-1, j \equiv 0,1, \ldots, M-1),
$$

которые содержат случайную помеху $\varepsilon_{k, j}$. В этом случае линейно-параметрическая дискретная модель (6), (7) принимает вид стохастических разностных уравнений:

$$
\begin{gathered}
z_{0,0}=\varepsilon_{0,0}, \quad z_{1,0}=\lambda_{7}+\varepsilon_{1,0} \\
z_{k, 0}=\lambda_{1} z_{k-1,0}+\lambda_{2} z_{k-2,0}+\lambda_{3}-\lambda_{1} \varepsilon_{k-1,0}-\lambda_{2} \varepsilon_{k-2,0}+\varepsilon_{k, 0}(k \equiv 2,3, \ldots, N-1), \\
z_{k, j}=\lambda_{4} z_{k, j-1}-\lambda_{4} \varepsilon_{k, j-1}+\varepsilon_{k, j}(k \equiv 0,1 ; j \equiv 1,2, \ldots, M-1) \\
z_{k, j}=\lambda_{1} z_{k-1, j}+\lambda_{2} z_{k-2, j}+\lambda_{4} z_{k, j-1}+\lambda_{5} z_{k-1, j-1}+\lambda_{6} z_{k-2, j-1}- \\
-\lambda_{1} \varepsilon_{k-1, j}-\lambda_{2} \varepsilon_{k-2, j}-\lambda_{4} \varepsilon_{k, j-1}-\lambda_{5} \varepsilon_{k-1, j-1}-\lambda_{6} \varepsilon_{k-2, j-1}+\varepsilon_{k, j} \\
\quad(k=2,3, \ldots, N-1 ; \quad j=1,2, \ldots, M-1) .
\end{gathered}
$$

Для оценки параметров этой модели можно использовать методы прикладного регрессионного анализа. В этом случае совокупность стохастических разностных уравнений следует представить в форме обобщённой регрессионной модели

$$
\left\{\begin{array}{l}
B=F \lambda+H, \\
H=\left(\Sigma-\lambda_{4} \Sigma_{1}\right) P_{\lambda} E,
\end{array}\right.
$$

элементы которой имеют блочную структуру: $B=\left[b_{1}\left|b_{2}\right| \cdots \mid b_{M}\right]^{\top}$-матрица-столбец размера $(N M \times 1)$, где $b_{j}=\left[z_{0, j-1}, z_{1, j-1}, \ldots, z_{N-1, j-1}\right]^{\top} \in \mathbb{R}^{N}$ $(j=1,2, \ldots, M) ; F=\left[F_{1}\left|F_{2}\right| \cdots \mid F_{M}\right]^{\top}$ - матрица размера $(N M \times 7)$, где

$$
\begin{gathered}
F_{1}=\left[\begin{array}{ccccccc}
0 & 0 & 0 & 0 & 0 & 0 & 0 \\
0 & 0 & 0 & 0 & 0 & 0 & 1 \\
z_{1,0} & z_{0,0} & 1 & 0 & 0 & 0 & 0 \\
z_{2,0} & z_{1,0} & 1 & 0 & 0 & 0 & 0 \\
\vdots & \vdots & \vdots & \vdots & \vdots & \vdots & \vdots \\
z_{N-2,0} & z_{N-3,0} & 1 & 0 & 0 & 0 & 0
\end{array}\right], \\
F_{j}=\left[\begin{array}{ccccccc}
0 & \\
0 & 0 & 0 & z_{0, j-2} & 0 & 0 & 0 \\
z_{1, j-1} & z_{0, j-1} & 0 & z_{2, j-2} & z_{1, j-2} & z_{0, j-2} & 0 \\
z_{2, j-1} & z_{1, j-1} & 0 & z_{3, j-2} & z_{2, j-2} & z_{1, j-2} & 0 \\
\vdots & \vdots & \vdots & \vdots & \vdots & \vdots & \vdots \\
z_{N-2, j-1} & z_{N-3, j-1} & 0 & z_{N-1, j-2} & z_{N-2, j-2} & z_{N-3, j-2} & 0
\end{array}\right]
\end{gathered}
$$

$(j \equiv 2,3, \ldots, M)$ - матрицы размера $(N \times 7) ; \lambda=\left[\lambda_{1}, \lambda_{2}, \lambda_{3}, \lambda_{4}, \lambda_{5}, \lambda_{6}, \lambda_{7}\right]^{\top}-$ вектор-столбец неизвестных коэффициентов регрессионной модели; $H=\left[H_{1} \mid\right.$ $\left.H_{2}|\cdots| H_{M}\right]^{\top}$ - матрица-столбец размера $(N M \times 1)$, в котором элементы векторов $H_{j}=\left(\eta_{0, j-1}, \eta_{1, j-1}, \ldots, \eta_{2, j-1}\right)^{\top} \in \mathbb{R}^{N}(j \equiv 1,2, \ldots, M)$ описываются 
следующими формулами:

$$
\begin{aligned}
\eta_{0,0} & =\varepsilon_{0,0} \\
\eta_{1,0} & =\varepsilon_{1,0} \\
\eta_{2,0} & =\varepsilon_{2,0}-\lambda_{2} \varepsilon_{0,0}-\lambda_{1} \varepsilon_{1,0} \\
\eta_{3,0} & =\varepsilon_{3,0}-\lambda_{2} \varepsilon_{1,0}-\lambda_{1} \varepsilon_{2,0} \\
& \vdots \\
\eta_{N-1,0} & =\varepsilon_{N-1,0}-\lambda_{2} \varepsilon_{N-3,0}-\lambda_{1} \varepsilon_{N-2,0}
\end{aligned}
$$

$$
\begin{aligned}
\eta_{0, j} & =\varepsilon_{0, j}-\lambda_{4} \varepsilon_{0, j-1} \\
\eta_{1, j} & =\varepsilon_{1, j}-\lambda_{4} \varepsilon_{1, j-1} \\
\eta_{2, j} & =\varepsilon_{2, j}-\lambda_{4}\left(-\lambda_{2} \varepsilon_{0, j-1}-\lambda_{1} \varepsilon_{1, j-1}+\varepsilon_{2, j-1}\right)-\lambda_{2} \varepsilon_{0, j}-\lambda_{1} \varepsilon_{1, j} \\
\eta_{3, j} & =\varepsilon_{3, j}-\lambda_{4}\left(-\lambda_{2} \varepsilon_{1, j-1}-\lambda_{1} \varepsilon_{2, j-1}+\varepsilon_{3, j-1}\right)-\lambda_{2} \varepsilon_{1, j}-\lambda_{1} \varepsilon_{2, j} \\
& \vdots \\
\eta_{N-1, j} & =\varepsilon_{N-1, j}-\lambda_{4}\left(-\lambda_{2} \varepsilon_{N-3, j-1}-\lambda_{1} \varepsilon_{N-2, j-1}+\varepsilon_{N-1, j-1}\right)-\lambda_{2} \varepsilon_{N-3, j}-\lambda_{1} \varepsilon_{N-2, j},
\end{aligned}
$$

где $j \equiv 2,3, \ldots, M-1$;

$$
P_{\lambda}=\left[\begin{array}{cccc}
P_{1} & \Theta & \ldots & \Theta \\
\Theta & P_{1} & \ldots & \Theta \\
\vdots & \vdots & \ddots & \vdots \\
\Theta & \Theta & \ldots & P_{1}
\end{array}\right], \Sigma=\left[\begin{array}{cccc}
I & \Theta & \ldots & \Theta \\
\Theta & I & \ldots & \Theta \\
\vdots & \vdots & \ddots & \vdots \\
\Theta & \Theta & \ldots & I
\end{array}\right], \Sigma_{1}=\left[\begin{array}{ccccc}
\Theta & \Theta & \ldots & \Theta & \Theta \\
I & \Theta & \ldots & \Theta & \Theta \\
\Theta & I & \ldots & \Theta & \Theta \\
\vdots & \vdots & \ddots & \vdots & \vdots \\
\Theta & \Theta & \ldots & I & \Theta
\end{array}\right]
$$

- блочные матрицы размера $(N M \times N M)$, в которых $I$ и $\Theta$ - единичная и нулевая матрицы размера $(N \times N)$, а матрица $P_{1}$ размера $(N \times N)$ имеет вид

$$
P_{1}=\left[\begin{array}{cccccccc}
1 & 0 & 0 & 0 & \ldots & 0 & 0 & 0 \\
0 & 1 & 0 & 0 & \ldots & 0 & 0 & 0 \\
-\lambda_{2} & -\lambda_{1} & 1 & 0 & \ldots & 0 & 0 & 0 \\
0 & -\lambda_{2} & -\lambda_{1} & 1 & \ldots & 0 & 0 & 0 \\
\vdots & \vdots & \vdots & \vdots & \ddots & \vdots & \vdots & \vdots \\
0 & 0 & 0 & 0 & \ldots & -\lambda_{2} & -\lambda_{1} & 1
\end{array}\right]
$$

Очевидно, что матрицы $P_{1}$ и $\Sigma-\lambda_{4} \Sigma_{1}$ невырожденные. Следовательно, существуют обратные матрицы $P_{\lambda}^{-1}$ и $\left(\Sigma-\lambda_{4} \Sigma_{1}\right)^{-1}$, где

$$
P_{\lambda}=\left[\begin{array}{ccccc}
P_{1}^{-1} & \Theta & \Theta & \ldots & \Theta \\
\Theta & P_{1}^{-1} & \Theta & \ldots & \Theta \\
\Theta & \Theta & P_{1}^{-1} & \ldots & \Theta \\
\vdots & \vdots & \vdots & \ddots & \vdots \\
\Theta & \Theta & \Theta & \ldots & P_{1}^{-1}
\end{array}\right]
$$

причём можно показать, что справедливо равенство

$$
\left(\Sigma-\lambda_{4} \Sigma_{1}\right)^{-1}=\Sigma+\lambda_{4} \Sigma_{1}+\lambda_{4}^{2} \Sigma_{1}^{2}+\ldots+\lambda_{4}^{M-1} \Sigma_{1}^{M-1} .
$$


Элементы обратной матрицы $P_{1}^{-1}$ описываются формулами [1]

$$
p_{i, j}^{-1}=\left\{\begin{array}{cccc}
0, & i<j, & i \equiv 1,2, \ldots, N, \quad i=2, j=1 \\
1, & i=j, & i \equiv 1,2, \ldots, N ; \\
\lambda_{1} p_{i-1, j}^{-1}+\lambda_{2} p_{i-2, j}^{-1}, & i>j, & i \equiv 3,4, \ldots, N, \quad j \equiv 1,2, \ldots, N .
\end{array}\right.
$$

$\mathrm{B}$ основе определения параметров $c_{1}, c_{2}, \alpha_{1}, \alpha_{2}, \beta$ пространственно-временной функциональной зависимости лежит среднеквадратичное оценивание коэффициентов $\lambda_{j}$ обобщённой регрессионной модели (8). Оценки коэффициентов обобщённой регрессионной модели находятся из условия минимизации среднеквадратичного отклонения построенной модели пространственновременной функциональной зависимости $\hat{z}(t, x)$ от экспериментальных данных $z_{k, j}$ :

$$
\|\hat{z}(t, x)-z(t, x)\|^{2}=\|\hat{\varepsilon}\|^{2} \rightarrow \min .
$$

С этой целью первое уравнение в (8) преобразуется к виду

$$
P_{\lambda}^{-1}\left(\Sigma-\lambda_{4} \Sigma_{1}\right)^{-1} B=P_{\lambda}^{-1}\left(\Sigma-\lambda_{4} \Sigma_{1}\right)^{-1} F \lambda+E .
$$

В этом случае задача сводится к минимизации функционала

$$
\|E\|^{2}=\left\|P_{\hat{\lambda}}^{-1}\left(\Sigma-\hat{\lambda}_{4} \Sigma_{1}\right)^{-1} B-P_{\hat{\lambda}}^{-1}\left(\Sigma-\hat{\lambda}_{4} \Sigma_{1}\right)^{-1} F \hat{\lambda}\right\|^{2} \rightarrow \min .
$$

Решение этой задачи на основе итерационной процедуры позволяет практически устранить смещение в оценках и тем самым добиться высокой точности результатов вычисления оценок параметров пространственно-временной функциональной зависимости [1].

Итерационная процедура среднеквадратичного оценивания коэффициентов разностной схемы описывается рекуррентными соотношениями

$$
\begin{gathered}
\hat{\lambda}^{(k+1)}=\left(F^{\top} \Omega_{\hat{\lambda}^{(k)}}^{-1} F\right)^{-1} F^{\top} \Omega_{\hat{\lambda}^{(k)}}^{-1} B \\
\Omega_{\hat{\lambda}^{(k)}}=\left(\Sigma-\hat{\lambda}_{4}^{(k)} \Sigma_{1}\right) P_{\hat{\lambda}^{(k)}} P_{\hat{\lambda}^{(k)}}^{\top}\left(\Sigma-\hat{\lambda}_{4}^{(k)} \Sigma_{1}\right)^{\top}, \quad k \equiv 0,1,2, \ldots .
\end{gathered}
$$

При этом за начальное приближение принимается $\hat{\lambda}^{(0)}=\left(F^{\top} F\right)^{-1} F^{\top} B$.

Найденные среднеквадратичные оценки $\hat{\lambda}_{j}$ коэффициентов обобщённой регрессионной модели (8) лежат в основе вычисления параметров двумерной функциональной зависимости $\tilde{z}(t, x)$. Сначала, в соответствии с формулами $(4)$, из решения квадратного уравнения $\hat{\lambda}_{2} \mu^{2}+\hat{\lambda}_{1} \mu-1=0$ находятся оценки $\hat{\mu}_{1}$ и $\hat{\mu}_{2}$. Затем по формулам вычисляются оценки параметров $\hat{\alpha}_{1}, \hat{\alpha}_{2}$ и $\hat{\beta}$ :

$$
\hat{\alpha}_{1}=\frac{1}{\tau} \ln \hat{\mu}_{1}, \quad \hat{\alpha}_{2}=\frac{1}{\tau} \ln \hat{\mu}_{2}, \quad \hat{\beta}=\frac{1}{h} \ln \hat{\lambda}_{4} .
$$

На заключительном этапе вычисляются оценки коэффициентов $c_{1}$ и $c_{2}$ из решения системы линейных алгебраических уравнений

$$
\left\{\begin{array}{l}
\hat{c}_{1}\left(1-\hat{\lambda}_{1}-\hat{\lambda}_{2}\right)+\hat{c}_{2}\left(1-\hat{\lambda}_{1}-\hat{\lambda}_{2}\right)=\hat{\lambda}_{3} \\
\hat{c}_{1}\left(1-\frac{1}{\hat{\mu}_{1}}\right)+\hat{c}_{2}\left(1-\frac{1}{\hat{\mu}_{2}}\right)=\hat{\lambda}_{7}
\end{array}\right.
$$


а именно $\hat{c}_{1}=\frac{\hat{\lambda}_{3}\left(1-\frac{1}{\hat{\mu}_{2}}\right)-\hat{\lambda}_{7}\left(1-\hat{\lambda}_{1}-\hat{\lambda}_{2}\right)}{\left(1-\hat{\lambda}_{1}-\hat{\lambda}_{2}\right)\left(\frac{1}{\hat{\mu}_{1}}-\frac{1}{\hat{\mu}_{2}}\right)}, \hat{c}_{2}=\frac{\hat{\lambda}_{7}\left(1-\hat{\lambda}_{1}-\hat{\lambda}_{2}\right)-\hat{\lambda}_{3}\left(1-\frac{1}{\hat{\mu}_{1}}\right)}{\left(1-\hat{\lambda}_{1}-\hat{\lambda}_{2}\right)\left(\frac{1}{\hat{\mu}_{1}}-\frac{1}{\hat{\mu}_{2}}\right)}$.

Проведены численно-аналитические исследования эффективности разработанного численного метода определения параметров двумерных пространственно-временных функциональных зависимостей на основе среднеквадратичного оценивания коэффициентов разностной схемы. Моделирование пространственно-временной функциональной зависимости (1) проводилось при следующих параметрах двумерного динамического процесса: $c_{1}=0,5, c_{2}=$ $=1,0, \alpha_{1}=2,0, \alpha_{2}=10,0, \beta=3,0$ и параметрах формирования результатов наблюдений: $N=10, M=4, \tau=0,111, h=0,1$. K смоделированным дискретным значениям двумерной функциональной зависимости

$$
\tilde{z}(t, x)=\left[c_{1}\left(1-e^{-\alpha_{1} t}\right)+c_{2}\left(1-e^{-\alpha_{2} t}\right)\right] e^{\beta x}
$$

добавлялась аддитивная случайная помеха, величина которой

$$
\varepsilon=\sqrt{\frac{\sum_{k=0}^{N-1} \sum_{j=0}^{M-1} \varepsilon_{k, j}^{2}}{\sum_{k=0}^{N-1} \sum_{j=0}^{M-1} \tilde{z}_{k, j}^{2}}}
$$

составляла 0,03 величины от мощности основного сигнала. На рис. 2 точками представлены смоделированные экспериментальные данные $z_{k, j}=\tilde{z}_{k, j}+\varepsilon_{k, j}$ $(k \equiv 0,1, \ldots, 9, j \equiv 0,1,2,3)$, а кривыми $0-3$ - восстановленные на основе среднеквадратичного оценивания коэффициентов разностной схемы зависимости $\hat{z}\left(t, x_{j}\right)$ при соответствующих $j$.

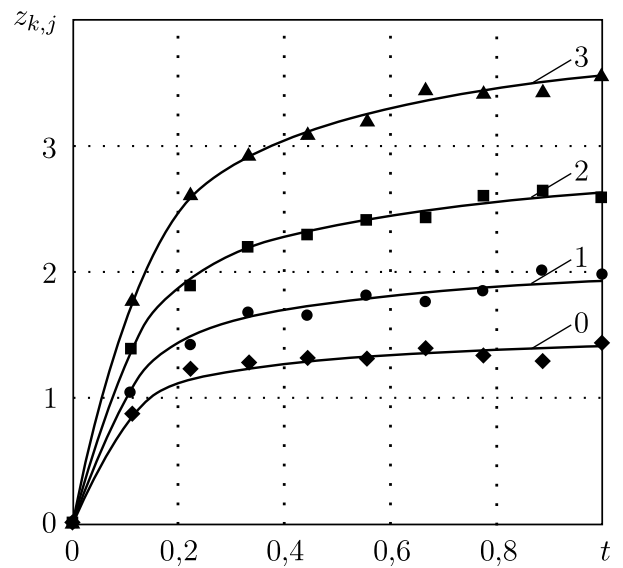

Рис. 2. Экспериментальные данные и восстановленная на основе среднеквадратичного оценивания коэффициентов разностной схемы двумерная пространственно-временная функциональная зависимость $\tilde{z}\left(t, x_{j}\right)$
Полученные результаты численно-аналитических исследований подтверждают высокую эффективность разработанного численного метода параметрической идентификации двумерных динамических систем на основе среднеквадратичного оценивания коэффициентов разностной схемы, описывающей результаты наблюдений. Предложенный подход к оценке параметров двумерных динамических процессов может быть использован в задачах параметрической идентификации широкого круга систем различной физической природы, например, в задачах построения детерминированных и стохастических моделей реологического деформирования материалов и элементов конструкций при первичной обработке серии экспериментальных кривых ползучести при постоянных напряжениях $[2,3]$, которые используются при решении соответствующих краевых задач $[4,5]$.

Работа выполнена при поддержке Федералъного агентства по образованию (проекть РНП 2.1.1/745 u РНП 2.1.1/3397). 


\section{БИБЛИОГРАФИЧЕСКИЙ СПИСОК}

1. Зотеев B. E. Параметрическая идентификация диссипативных механических систем на основе разностных уравнений / ред. В. П. Радченко. - М.: Машиностроение-1, 2009. $344 \mathrm{c}$.

2. Радченко В.П., Голудин Е.П. Феноменологическая стохастическая модель изотермической ползучести поливинилхлоридного пластиката // Becmн. Сам. гос. техн. ун-та. Сер. Физ.-мат. науки, 2008. - №1(16). - С. 45-52.

3. Радченко В. П., Еремин Ю. А. Реологическое деформирование и разрушение материалов и элементов конструкций. - М.: Машиностроение-1, 2004. - 265 с.

4. Коваленко Л. В., Попов Н.Н., Радченко В. П. Решение плоской стохастической краевой задачи ползучести // ПMM, 2009. - Т. 73, №6. - С. 1009-1016; англ. пер.: Kovalenko L. V., Popov N. N., Radchenko V.P. Solution of the plane stochastic creep boundary value problem // Journal of Applied Mathematics and Mechanics Vol.73, No.6. - Article in press.

5. Радченко В. П., Попов Н. Н. Нелинейная стохастическая задача ползучести неоднородной плоскости с учетом повреждённости материала // ПМТФ, 2007. — Т. 49, №3. C. 140-146; англ. пер.: Popov N. N., Radchenko V.P. Nonlinear stochastic creep problem for an inhomogeneous plane with the damage to the material taken into account // Journal of Applied Mechanics and Technical Physics, 2007. — Vol. 48, No. 2. — P. 265-270.

Поступила в редакцию 26/II/2010; в окончательном варианте - 3/III $/ 2010$.

MSC: 65P40, 34C15, 37M05

\section{DEFINITION OF PARAMETERS OF 2D DYNAMICAL PROCESSES ON THE BASIS OF DIFFERENCE SCHEMES}

\section{A. Zausaeva, V.E. Zoteev}

Samara State Technical University,

244, Molodogvardeyskaya str., Samara, 443100.

E-mail: zausmasha@mail.ru

The construction of difference schemes, describing the results of observations of $2 D$ spatio-temporal functional dependencies and a numerical method for definition of the parameters of such dependencies on the basis of difference schemes are studied. The algorithm of method including iteration procedure for mean-square estimation of coefficients of linear parametric discrete model in the form of stochastic difference equations. Such an approach to solving the problem of identification of $2 D$ dynamic processes can ensure a high adequacy of a model, and as a consequence, to achieve high accuracy of estimating the parameters of the model.

Key words: parametrical identification, difference scheme, root-mean-square approximation.

Original article submitted 26/II/2010; revision submitted $3 / \mathrm{III} / 2010$.

Maria A. Zausaeva, Postgraduate Student, Dept. of Applied Mathematics \& Computer Science. Vladimir E. Zoteev (Ph.D. (Phys. \& Math.)), Associate Professor, Dept. of Applied Mathematics \& Computer Science. 